\title{
Inverse Modelling in Geology by Interactive Evolutionary Computation
}

\author{
Chris Wijns ${ }^{\mathrm{a}, \mathrm{b}, *}$, Fabio Boschetti ${ }^{\mathrm{a}}$, Louis Moresi ${ }^{\mathrm{a}, \mathrm{c}}$

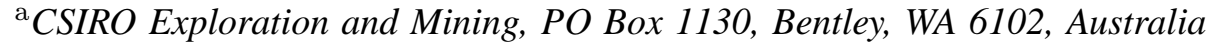 \\ ${ }^{\mathrm{b}}$ Centre for Global Metallogeny, University of Western Australia, Crawley, WA 6009, \\ Australia \\ ${ }^{\mathrm{c}}$ now at School of Mathematical Sciences, Monash University, Building 28, VIC 3800, \\ Australia
}

\begin{abstract}
Inverse modelling of geological processes, in the absence of established numerical criteria to act as inversion targets, requires an approach which uses human interaction to assess forward model results. The method of interactive evolutionary computation provides for the inclusion of qualitative geological expertise within a rigorous mathematical inversion scheme, by simply asking an expert user to evaluate a sequence of forward geological models. The traditional numerical misfit is replaced by a human appraisal of misfit. We use this interactive technique to successfully invert a geodynamic model for a conceptual pattern of fault spacing during crustal extension.
\end{abstract}

Key words: inverse modelling, interactive evolutionary computation, genetic algorithm, faulting

PACS: 07.05.Mh, 07.05.Tp, 02.50.Sk

\section{Introduction}

In recent years, powerful computers have allowed the development of quite sophisticated forward modelling of geological processes. Subduction, faulting and folding, mantle convection, and fluid flows can all be treated in a rigorous numerical fashion, much in the same way as traditional geophysical applications such as seismic or potential field problems.

\footnotetext{
* Corresponding author.
}

Email address:

cwijns@geol. uwa. edu. au (Chris Wijns).
As modelling capabilities have increased, so has the number of parameters which enter into the model, and this has lead to the increasingly difficult task of sorting out the influence of these parameters. In the nonlinear geological systems which we investigate today, predicting forward model behaviour through a knowledge of individual parameter influences may often be unfeasible.

In general, forward modelling allows us to answer questions such as "What response should I expect from this distribution of 
material properties under these initial conditions?" (e.g. "What faults will be generated by this stress field in this particular material?"). The answer is obtained by providing a computer code with certain input parameters and running the code for a number of time steps. In many cases the final result is displayed as a geological section or $3 \mathrm{D}$ model.

Most real geological problems require an answer to a question that goes in the opposite direction, i.e., "What combination of material properties and initial conditions may result in this geological response?" (e.g. "What was the stress field which generated these faults in this rock?"). This is a more complicated problem, for which the answer must be found by iterative numerical trial and error methods. This is computationally intensive, and the manner in which we optimise the search for an answer is called inverse theory, or simply inversion (Tarantola, 1987).

Inversion is the natural step forward in geological modelling. Reconstructing initial configurations from their geological responses is very much what geology is about. It is an implicit inverse problem tackled on a daily basis by every geologist. The basic difficulties encountered in an inverse problem are the lack of a guaranteed solution, or the probable existence of many solutions giving the same answer (non-uniqueness), the efficiency with which a solution can be found that matches a target within a given tolerance, and the sensitivity of a given solution to changes in the initial conditions. For the mathematical and technical aspects of inversion we refer the reader to Parker (1977) or Tarantola (1987).

To overcome the difficulties of inversion, geologists use their intuition and experience to focus only on the "geologically reasonable" models which lead to the particular features they observe. This qualitative knowledge is often difficult to quantify, and we end up with conceptual geological targets which cannot be adequately described by numerical data. This is in contrast to, for example, the inversion of a gravity profile, where the gravity data are the objective measure we are trying to reproduce. To compensate for the lack of a quantitative target, we have combined the formal methodology of mathematical inversion with the knowledge held in observational experience. The inverse modelling technique we propose can help every time a problem needs visual appraisal of the results or a priori expert knowledge. All that is required is a code that allows the user to forward model a process and view its result.

\section{Method}

At present, geodynamic modelling is often confined to the forward modelling stage. Success has been achieved in quantitative inversion of sedimentary basin models (e.g. Cross and Lessenger, 1999; Bellingham and White, 2000), demonstrating cases where available quantitative data (i.e. borehole logs and stratigraphic horizons from seismic interpretations) can be used for a direct measure of misfit. Kaus and Podladchikov (2001) were able to inverse model a Rayleigh-Taylor instability to restore initial conditions, but only for very restricted cases of initial geometry. In many applications of geological modelling, a forward solution is judged visually according to its resemblance to patterns seen in the field, to the fact that it does not contradict basic geological principles, or simply according to the modeller's expectations.

If we accept the fact that much of a geologist's expertise is difficult to quantify, then we need a method to incorporate human in- 
teraction in directing the inversion process. Recently, research in artificial intelligence has resulted in systems to support such human interaction in optimisation problems (Takagi, 2001). They have been used in such diverse fields as graphic design, music composition, and the engineering of hearing aids. These systems are known collectively under the term interactive evolutionary computation (IEC).

We have extended the use of IEC to geological applications in which visual judgment is necessary to evaluate model results in the absence of sufficient constraints. The system represents an advance on traditional, timeconsuming trial and error approaches by providing a formal role in the inversion process for geological experience which cannot be transformed into data. The traditional numerical measure of data mismatch is replaced by the user's subjective evaluation. Humans find it hard to express subjective judgment with absolute values, while they generally find it much easier to compare different instances of the same process and rank them according to certain criteria. Consequently, interactive inversion works by producing different possible solutions and presenting them to the user for judgment and ranking.

Genetic algorithms (GAs) are one search method suitable for the inversion of highly non-linear functions. Starting with a set of random solutions, these algorithms progressively modify the solution set by imitating the evolutionary behavior of biological systems (selection, cross-over, and mutation), until an acceptable result is achieved. GAs belong to a class of algorithms that work by optimising an ensemble of solutions, unlike other classes that optimise one single solution. They are also suitable for handling ranked suites of solutions, which makes them an obvious choice as the internal engine for interactive inversion applications.

GAs are an established technique today, with a wide range of applications to both theoretical and industrial problems. We refer the reader to Goldberg (1989) for a basic description of GAs, and to Boschetti et al. (1996) for a more detailed description of the specific GA implementation used in this work.

Our IEC system works by linking a geological forward model to the GA. The inversion process is as follows: a geologist runs the forward-modelling code with the aim of producing a geological model that matches a conceptual target. A number of selected parameters is allowed to vary within given ranges. The GA initially generates a suite of different models using randomly picked parameter values. These models could be static geological models or animations showing time evolution. In the example we present below, we have no automated method for discriminating between geologically appropriate results, so the geologist ranks each of them according to criteria founded in his or her experience and knowledge. A relative target misfit is now contained within these rankings. When this stage is complete, the GA applies parameter swapping between highly-ranked models, to generate a new set of models that progressively converges towards the target geological section. As in biological evolution, an element of randomness exists in the generation of new models, so that unexpected results may suggest new possibilities outside the experience or expectation of the geologist. 


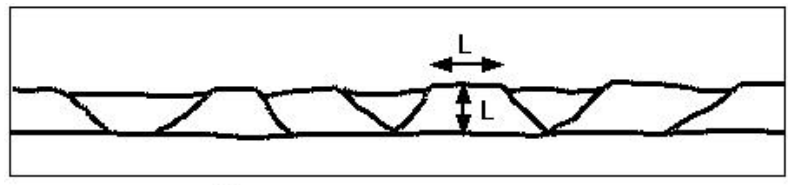

a

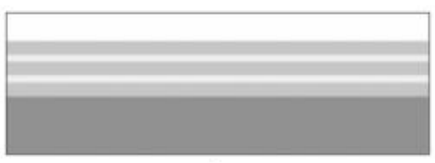

b

Fig. 1. (a) Conceptual inversion target showing faults spaced on the order of the thickness of the upper crust. (b) Initial geometry of the numerical model. The upper crust contains light-coloured marker units with no material differences.

\section{Application}

\subsection{Model}

We illustrate the inversion process using an example of faulting during crustal extension. The goal is to find a set of material parameters which gives rise to fault spacing on the order of the thickness of the upper crust, while nowhere enabling the upper crust to completely pull apart. The forward modelling code we have chosen to link to the IEC method is a particle-in-cell finite element code which is well-suited to problems involving very large deformation (Moresi et al., 2001, 2002). The results of forward numerical models are ranked by comparing them to the simplified line sketch of Fig. 1(a). It is important to note that the method can proceed without any actual target image. The target is included as a guide, and we are not trying to reproduce the exact number, location, and dip of the faults which are drawn. In fact, the location of faults is extremely sensitive to initial perturbations, both in nature and in our numerical models, so that it is all the more appropriate to look for general behaviour and relative spacing.
The model is composed of two initially homogeneous crustal layers. The upper layer behaves as a visco-plastic material, and the bottom layer has a Newtonian viscosity. On top of these is a low density, low viscosity background material ("air") which does not interfere with the mechanics of the problem. The fact that the mantle is not included is akin to specifying a strong mantle which does not appreciably deflect during extension. This initial configuration is illustrated in Fig. 1(b). Light-coloured horizons in the upper crust are simply marker units with no physical differences. True dimensions, material parameters, and scaling methods are explained below. The upper crust has strain-weakening properties, which cause initial strain perturbations to localise. The fault geometry and successive fault spacing arise naturally from the initial conditions of the problem. Strainweakening is our approximation to brittle behaviour in the upper crust - a yield law prescribes an upper limit $\sigma_{y}$ on the stress according to

$$
\sigma_{y}=\left(B_{o}+B_{p} p\right) f(\varepsilon)
$$

where $p$ is the pressure, $B_{o}$ is the cohesion, or yield stress at zero pressure, and $B_{p}$ is the pressure dependence of the yield stress, equivalent to the friction coefficient in Byerlee's law (Byerlee, 1968). Strain-weakening is included through the power law function $f(\varepsilon)$, in which $\varepsilon$ is the accumulated plastic strain, measured as the second invariant of the deviatoric strain tensor.

$$
f(\varepsilon)= \begin{cases}1-\left(1-E_{a}\right)\left(\varepsilon / \varepsilon_{o}\right)^{E_{n}} & \varepsilon<\varepsilon_{o} \\ E_{a} & \varepsilon \geq \varepsilon_{o}\end{cases}
$$

The roles of the parameters $E_{a}, \varepsilon_{o}$, and $E_{n}$ are illustrated graphically in Fig. 2. The "saturation" strain $\varepsilon_{o}$ is the accumulated plastic strain beyond which no further weakening takes place, and at this point the maximum 


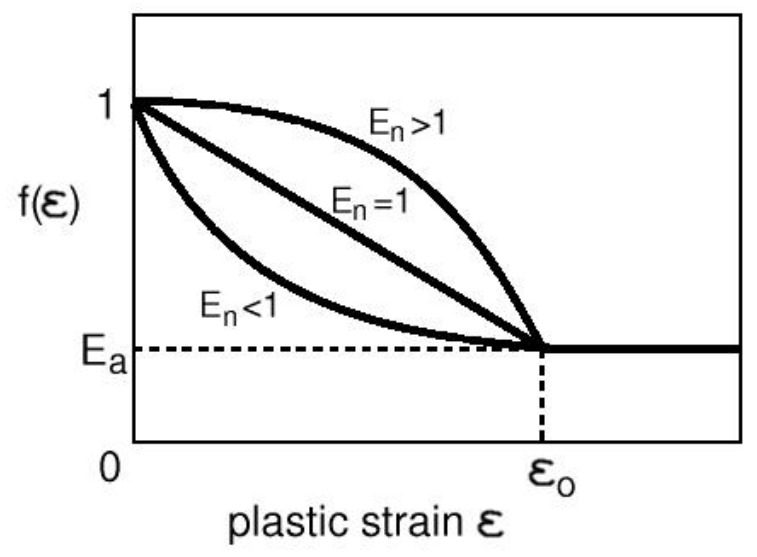

Fig. 2. The strain weakening function $f(\varepsilon)$ is determined by the ratio $E_{a}$ of minimum to original viscosity, the "saturation strain" $\varepsilon_{o}$ after which there is no further weakening, and the exponent $E_{n}$ which dictates the curvature of the weakening relation.

proportion of strain weakening is given by $E_{a} . E_{a}=1$ implies no strain weakening. The exponent $E_{n}$ describes the shape of the decay curve. The manner in which faults actually weaken is poorly understood, so all three parameters are allowed to vary, in an attempt to gauge the influence of the weakening law. The fourth parameter for which we seek to quantify the effect is the viscosity of the lower crust. These variables are listed in Table 1, together with their ranges of investigation.

\subsection{Scaling}

Numerous studies of the Basin and Range area in the western U.S.A. provide data on an environment which most agree is extensional in nature, and so we adopt many of our parameter values from the literature for this region. The overall amount of extension is arguable, from a few tens of percent, to greater than $100 \%$ (e.g. Jones et al., 1992) in certain regions. We choose to compare structures after $50 \%$ extension. The initial $3 \times 1$ model box of Fig. 1(b) represents natural di- mensions of 150 by $50 \mathrm{~km}$. Thus the upper and lower crust are each $20 \mathrm{~km}$ thick initially, and after extension the total thickness of the crust is about $25 \mathrm{~km}$, which is representative of large areas in the Basin and Range, based on the regional crustal thickness model of Chulick et al. (2001). Comparison of model versus natural dimensions leads to a length scale factor

$$
h^{*}=\frac{h_{\text {model }}}{h_{\text {nature }}}=2 \times 10^{-5} .
$$

The rate of extension is taken from data typical of the western U.S.A. Based on GPS data through time, Thatcher et al. (1999) have determined velocities of between 2.8 and 6.5 $\mathrm{mm} / \mathrm{yr}$ for various domains of the Basin and Range, and Murray and Segall (2001) find extension rates of between 2.3 and $3.6 \mathrm{~mm} / \mathrm{yr}$. Over our similar length scale of $150 \mathrm{~km}$, $5 \mathrm{~mm} / \mathrm{yr}$ is equivalent to a strain rate $\dot{\varepsilon}=$ $1.06 \times 10^{-15} \mathrm{~s}^{-1}$. Comparison with our model strain rate of 0.33 gives a scale factor $\dot{\varepsilon}^{*}=$ $3.1 \times 10^{14}$.

Equivalence of lithostatic versus viscous stresses demands that

$$
\rho^{*} g^{*} h^{*}=\eta^{*} \dot{\varepsilon}^{*} .
$$

We have chosen model densities for the upper and lower crust of 2700 and $3000 \mathrm{~kg} / \mathrm{m}^{3}$, respectively, so that both the density scale $\rho^{*}$ and gravitational scale $g^{*}$ are equal to unity. The resultant viscosity scale factor $\eta^{*}=6.4 \times 10^{-20}$. We choose the viscosity $\eta_{u}$ of the upper crust in conjunction with $B_{p}$ such that the initial depth of maximum yield stress coincides with the interface $z_{u}$ between upper and lower crust.

$$
\sigma_{y}\left(z_{u}\right)=\eta_{u} \dot{\varepsilon}
$$

Using Eq. (1) for the yield stress before any strain-softening, and noting that the total pressure $p$ is the lithostatic stress reduced by 


\begin{tabular}{|c|c|c|c|c|}
\hline Parameter & Initial range & Increment & "Best" value & Range \\
\hline$\eta_{l}\left(\times 1.56 \times 10^{19} \mathrm{~Pa} \cdot \mathrm{s}\right)$ & $10-500$ & 25 & 400 & 400 \\
$E_{a}$ & $0.1-1.0$ & 0.1 & 0.6 & 0.6 \\
$\varepsilon_{o}$ & $0.0-1.0$ & 0.1 & 0.7 & $0.7-0.9$ \\
$E_{n}$ & $0.4-2.0$ & 0.2 & 1.0 & $0.6-1.0$ \\
\hline
\end{tabular}

Table 1

Four model parameters, described in the text, are free to vary during the inversion. The "best" values give rise to the top-ranked model of the final (sixth) generation. The last column gives the range of parameter values for the top five models of the final generation.

the extensional stress,

$$
\begin{aligned}
B_{o}+B_{p}\left(\rho g z_{u}-\eta_{u} \dot{\varepsilon}\right) & =\eta_{u} \dot{\varepsilon} \\
\eta_{u} & =\frac{B_{o}+\rho g z_{u} B_{p}}{\left(1+B_{p}\right) \dot{\varepsilon}} .
\end{aligned}
$$

We are left to choose values for the cohesion $B_{o}$ and the friction coefficient $B_{p}$. We choose a low rock cohesion of $10 \mathrm{MPa}$ (e.g. Suppe, $1985, p .155)$, which, relative to the stresses in our models, is close to the value of zero employed in Byerlee's law for the upper 10 $\mathrm{km}$ of the crust. Numerous laboratory experiments by Byerlee (1968) resulted in a universal friction coefficient of $0.6-0.85$ for most rock types. However, this is for dry samples. Assuming an average hydrostatic pore pressure, the effective pressure is reduced by more than one third, so that an equivalent dry friction coefficient of 0.7 is reduced to 0.44 . Using these values of $B_{o}$ and $B_{p}$, Eq. (2) gives an effective upper crust viscosity of about $10^{23} \mathrm{~Pa} \cdot \mathrm{s}$.

\subsection{Inversion}

Six forward models are run at each step of the inversion, and the four parameters in Table 1 are allowed to vary. Initially, these parameter values are chosen randomly by the
GA, within the bounds specified. Extension proceeds by applying a uniform velocity to the right-hand boundary. Figs. 3, 4, and 5 illustrate the evolution of results using the IEC algorithm. We infer that bands of high localised plastic strain represent faults. Accumulated strain in the upper crust is indicated by darkened material, and the degree of shading is indicative of the amount of strain. The first generation (Fig. 3) produces no satisfactory model. The image ranked first has the most desirable fault spacing out of the choices presented, and also exhibits the most clearly-defined faults. The third and fourth models are ranked in the bottom because they have resulted in complete dissection of the upper crust, which, as mentioned above, we are explicitly avoiding in this example. The GA now uses these rankings as a measure of relative misfit.

Fig. 4 contains the second generation of the inversion. From this generation onward, we always compare results with the best-ranked model of the previous generation, in order to assess whether we are converging towards the target. In this example, there are no secondgeneration models which have improved upon the best first- generation model. This is still a legitimate and useful result which tells the GA that it has gone exploring parameter space in the wrong direction. However, over- 


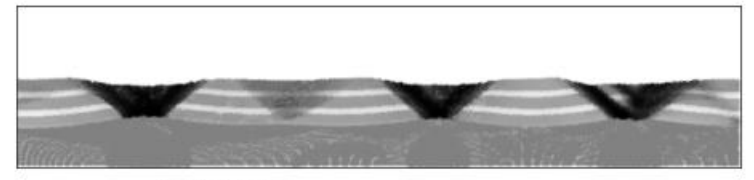

Rank: 2

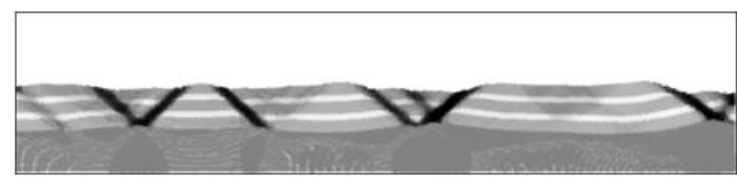

Rank: 1

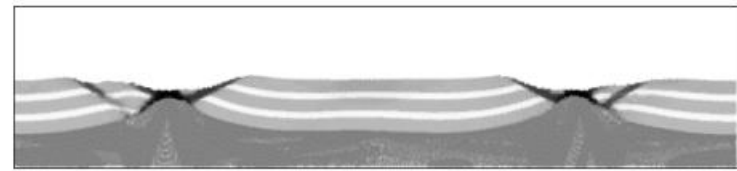

Rank: 5

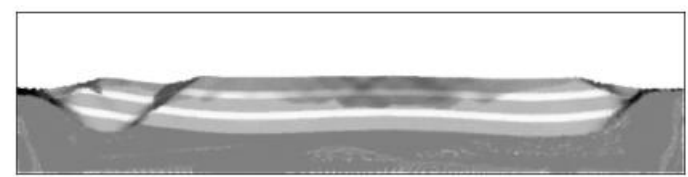

Rank: 6

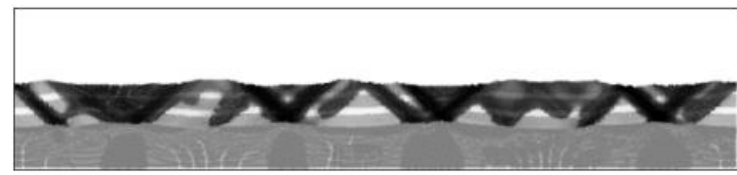

Rank: 3

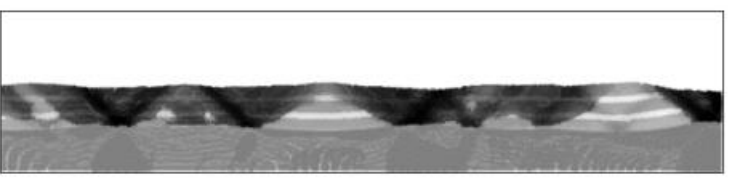

Rank: 4

Fig. 3. First generation of the inversion process. Dark areas in the upper crust represent accumulated plastic strain. Model results are ranked according to their similarity to the concepts embodied in the target image of Fig. 1. (Faint white lines in the lower crust represent uneven particle distribution after deformation, and are not a visualisation of any physical process. They are interference patterns between the deformed distribution of particles and the plotting routine which allocates colours to a regular array of pixels based on nearby particles. Although particles reproduce in order to accomodate minimum requirements for computation, they do not necessarily reproduce sufficiently for aesthetic purposes.)

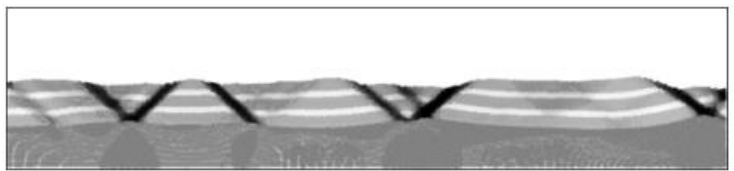

Rank: 1

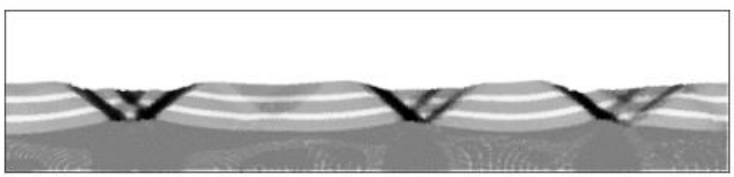

Rank: 7

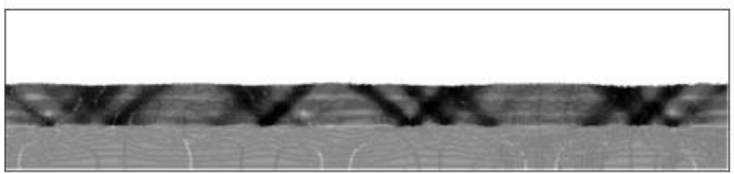

Rank: 4

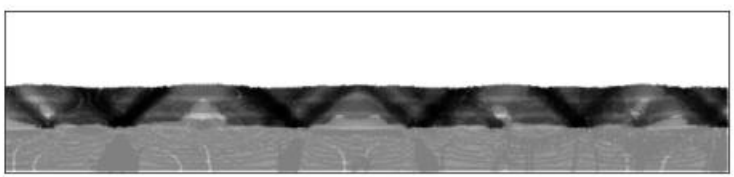

Rank: 3

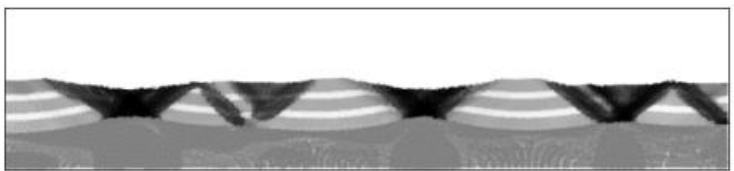

Rank: 5

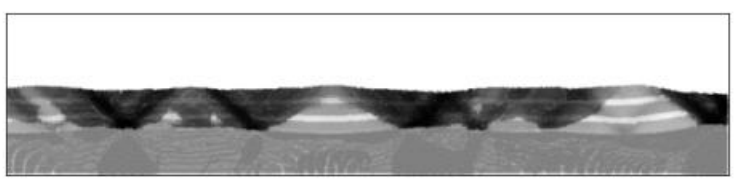

Rank: 6

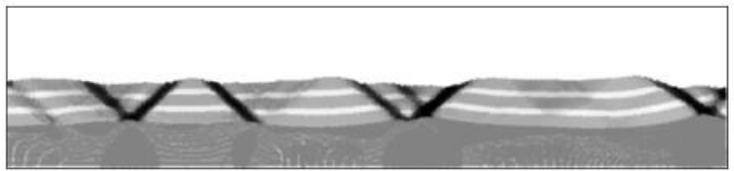

Rank: 2 (best of gen. 1)

Fig. 4. Second generation of the inversion process. Results are compared with the best-ranked model of the first generation.

all results are better in the sense that we no longer have any models with dissected upper crust. The model ranked third was so chosen in order to encourage denser fault spacing.

We continue iterating the ranking process a total of six times, at which point five of 


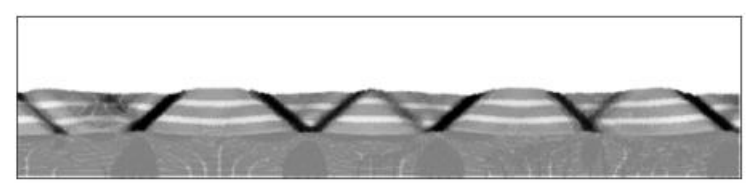

Rank: 4

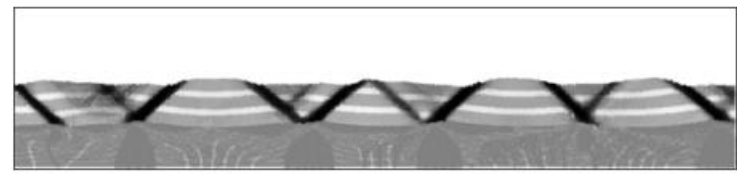

Rank: 1

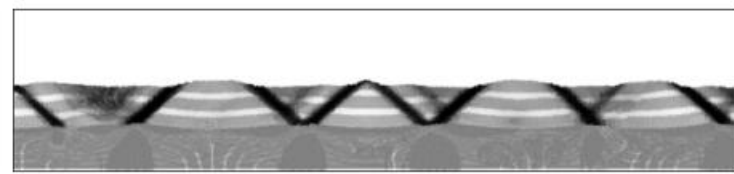

Rank: 3

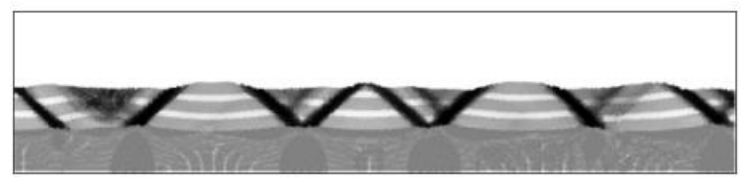

Rank: 5

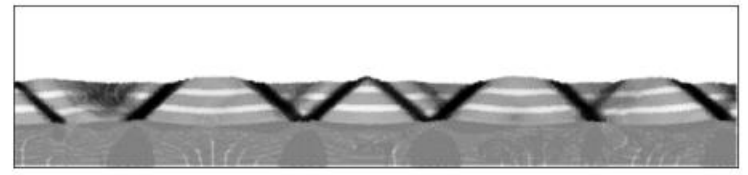

Rank: 2

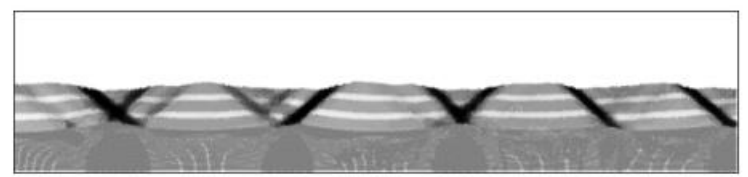

Rank: 7

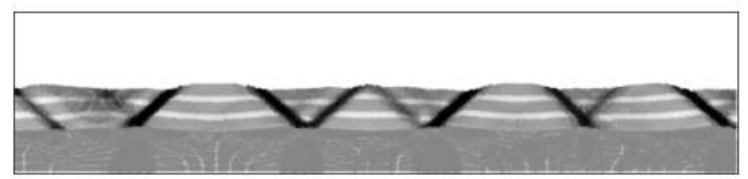

Rank: 6 (best of gen. 5)

Fig. 5. Sixth and final generation of the inversion process. Almost all models are qualitatively similar, with no substantial improvement over the best-ranked model of the previous generation.

the resulting images are qualitatively similar (Fig. 5), and show no evidence of substantial improvement over the best-ranked model of generation five. It is in fact difficult to assign different rankings to the first five images. The models have a regular fault spacing which corresponds to our target idea. It is apparent that although the features of the final models are satisfactory in concept, they would not match up well with the target image through any quantitative direct image comparison.

The outcome of this experiment is a set of our four chosen parameters that leads to the qualitative behaviour which we targeted. Table 1 lists the final ("best") values which give rise to the highest-ranked model of the sixth and final generation (Fig. 5, second model). We also show the range of each parameter for the five top-ranked models of the final generation, as a measure of variability within visually equivalent results. We can draw a few conclusions from these final values. The lower crust has an optimal viscosity which is about $6.2 \times 10^{21} \mathrm{~Pa} \cdot \mathrm{s}$. This is about one order of magnitude greater than the upper limit found by Pollitz et al. (2001), based upon geodetic measurements of post-seismic velocity fields after the 1999 Hector Mine earthquake in California. Flesch et al. (2000) find a $100 \mathrm{~km}$ vertically averaged crustal viscosity of between $5 \times 10^{21}$ and $5 \times 10^{22} \mathrm{~Pa} \cdot \mathrm{s}$ for most of the western U.S.A. Depending on mantle viscosity and the method of vertical averaging, our result may lie within this range. Our means of calculating an effective viscosity for the upper crust influences the result for the lower crust, so that rather than discuss absolute viscosity values, it would be fairer to state that the optimal fault spacing occurs when the lower crust is about one fifteenth the strength of the upper crust. Upon examination of all models, we observe that smaller viscosities for the lower crust lead to dissection of the upper crust (models 3 and 4 of generation 1) and metamorphic core complex formation. Higher viscosities lead to more even stretching of the upper crust and probably finely-spaced faulting which is below the resolution of the present modelling (e.g. model 3 of generation 2). The results for the strain- 
weakening law settle on a value of $E_{a}$ which reflects a yield strength of $60 \%$ of the original strength after a total strain accumulation $\varepsilon_{o}$ of 0.7 to 0.9 . Major faults may undergo significantly more weakening than this, according to both numerical experiments (e.g. Bird and Kong, 1994), and field-based heat-flow measurements (e.g. Lachenbruch and Sass, 1980, 1992). The fact that our model faults weaken less may contribute to the phenomenon of distributed faulting, whereas greater weakening may promote a higher degree of localisation on single faults. Lastly, the exponent $E_{n}$ varies between 0.6 and 1.0, indicating that the shape of the weakening curve is less important than the other parameters in controlling fault spacing.

\section{Discussion}

In the above example, we wish to arrive at some particular behaviour of the crust during deformation - behaviour which we are not able to sufficiently describe by numerical measures. Finding a suitable combination of parameters which gives rise to this behaviour would previously have involved one of two more laborious approaches: the manual selection of parameters by trial and error, or an exhaustive coverage of all parametric space. Trial and error may succeed with a limited number of parameters, but depends upon the user's knowledge of the coupling and feedback between parameters, which, in highly non-linear problems involving complex crustal rheologies, may be impossible. A parametric sweep quickly becomes unfeasible due to the sheer number of models which must be run as the number of parameters is increased. In our example, in excess of 20 000 models would have to be run in order to cover all possible parameter combinations, and each forward model takes a few hours to run on a $935 \mathrm{MHz}$ desktop computer with $500 \mathrm{MB}$ of RAM. Using IEC, we have found multiple solutions with only 36 models being run. This vast reduction in the number of individual models can be attributed to the fact that visual ranking provides more information in this type of search than numerical misfit in a non-interactive inversion. A model containing one or more features of paramount importance, but with a potentially large numerical misfit because of, for example, spatial discrepancy in feature locations, is ranked highly and provides a significant step forward in the search through parameter space. In fact, because of the combinatorial nature of the GA progression, two images which each contain a different feature of importance can both be ranked highly in order to increase the likelyhood of producing a new model containing both desired features. Neither trial and error, nor a parametric sweep, takes full advantage of the expert knowledge of a user, which in this case is the observational experience of a field geologist.

Our IEC method can be used by a field expert to invert for model parameters through the comparison of suitable images. A visual target has replaced a numerical target, and relative ranking of model results provides the measure of misfit. The GA converges upon a set of parameters which allows us to reproduce the conceptual model of the geologist. In our example above, both the number of variable parameters, and the number of individual models in each generation, is limited by available computer speed. The number of models per generation would ultimately be limited by the capacity of a human to distinguish between and rank the results.

The power of inversion lies in demonstrating the range of non-uniqueness of a solution, and with this method we have performed a very simple and quick $a$ posteriori investiga- 
tion of the sensitivity of the results to changes in our chosen variables. For example, referring to Table 1 , the viscosity of the lower crust has settled on a unique value, which suggests that it exerts a strong influence on the outcome of the problem. The same is true of the final amount of strain weakening $E_{a}$. A parameter such as the saturation strain $\varepsilon_{o}$ has a weaker influence on the outcome, since it varies slightly without affecting the final qualitative picture. The shape of the strainweakening function, which is determined by $E_{n}$, has the least influence. With the inversion approach in general, most late-stage models are close to the target in parameter space, and so a back-analysis through all generations is instructive for looking at the sensitivity of solutions. Future work will concentrate on effective visualisation of the whole multidimensional parameter space, including better analysis of solution sensitivity and nonuniqueness.

An important component of this interactive inversion technique is its capability of embracing unforseen results. An intuitive approach relies entirely on the experience of the modeller, and may miss realistic targets which lie outside the realm of modelling space which is envisaged. The GA, although converging upon a specific area in parameter space, also provides for random solutions. If ranked highly, such a random solution may open up an entirely different class of models which also yield realistic results.

\section{Conclusions}

We have demonstrated the geological application of an inversion algorithm which uses visual comparison between solutions and a target as a proxy for numerical misfit, in situations where we have no appropriate numer- ical description of the target. The technique of interactive evolutionary computation has considerably diminished the effort required to explore parameter space during the inversion of conceptual models in geology. The method is particularly geared towards cases of highly non-linear interactions between material parameters, where resultant material behaviour is difficult to predict. We accomodate the lack of a numerical inversion target by using a genetic algorithm together with image ranking to focus on a visual target. This approach exploits the unquantifiable aspects of an expert user's knowledge in a situation where this is currently an under-utilised resource.

\section{Acknowledgements}

The authors thank Dr. Susan Ellis for insisting on a discussion of scaling and comparison with natural values, and Dr. Nicholas White for pointing out the research done on quantitative inversion in basin modelling.

\section{References}

Bellingham, P., White, N., 2000. A general inverse method for modelling extensional sedimentary basins. Basin Research 12, 219-226.

Bird, P., Kong, X., 1994. Computer simulations of California tectonics confirm very low strength of major faults. Geological Society of America Bulletin 106, 159-174.

Boschetti, F., Dentith, M., List, R., 1996. Inversion of seismic refraction data using genetic algorithms. Geophysics 61, 1715-1727.

Brace, W., Kohlstedt, D., 1980. Limits on lithospheric stress imposed by laboratory experiments. Journal of Geophysical Research 85, 6248-6252.

Byerlee, J. D., 1968. Brittle-ductile transition in rocks. Journal of Geophysical Research 73, 4741-4750. 
Chulick, G., Mooney, W. D., Detweiler, S., Nov. 2001. Seismic properties of North America and the surrounding ocean basins, http://quake.wr.usgs.gov/research/ structure/CrustalStructure/nam.

Cross, T., Lessenger, M., 1999. Construction and application of a stratigraphic inverse model. In: Harbaugh, J., Watney, W., Rankey, E., Slingerland, R., Goldstein, R., Franseen, E. (Eds.), SEPM Special Publication 62: Numerical Experiments in Stratigraphy: Recent Advances in Stratigraphic and Sedimentologic Computer Simulations. SEPM (Society for Sedimentary Geology), pp. 69-83.

Flesch, L., Holt, W., Haines, A., Shen-Tu, B., 2000. Dynamics of the Pacific-North American plate boundary zone in the western United States. Science 287, 834-836.

Goldberg, D., 1989. Genetic Algorithms in Search, Optimization, and Machine Learning. Addison-Wesley, Reading, Massachusetts, 412 pp.

Jones, C., Wernicke, B., Farmer, G., Walker, J., Coleman, D., McKenna, L. W., Perry, F. V., 1992. Variations across and along a major continental rift: An interdisciplinary study of the Basin and Range province, western USA. Tectonophysics 213, 57-96.

Kaus, B. J., Podladchikov, Y. Y., 2001. Forward and reverse modeling of the three-dimensional viscous Rayleigh-Taylor instability. Geophysical Research Letters 28, 11095-11098.

Lachenbruch, A., Sass, J., 1980. Heat flow and energetics of the San Andreas fault zone. Journal of Geophysical Research 85, 6185-6222.

Lachenbruch, A., Sass, J., 1992. Heat flow from Cajon Pass, fault strength, and tectonic implications. Journal of Geophysical Research 97, 4995-5015.

Moresi, L., Dufour, F., Mühlhaus, H.-B., 2002. Mantle convection models with viscoelastic/brittle lithosphere: Numerical methodology and plate tectonic modeling. Pure and Applied Geophysics 159, 2335-2356.

Moresi, L., Mühlhaus, H.-B., Dufour, F., 2001. Viscoelastic formulation for modelling of plate tectonics. In: Mühlhaus, H.-B., Dyskin, A., Pasternak, E. (Eds.), Bifurcation and Localiza- tion in Soils and Rocks. Balkema, Rotterdam, pp. 337-344.

Murray, M. H., Segall, P., 2001. Modeling broadscale deformation in northern California and Nevada from plate motions and elastic strain accumulation. Geophysical Research Letters 28, 4315-4318.

Parker, R., 1977. Understanding inverse theory. Annual Review of Earth and Planetary Sciences 5, 35-64.

Pollitz, F., Wicks, C., Thatcher, W., 2001. Mantle flow beneath a continental strike-slip fault: Postseismic deformation after the 1999 Hector Mine earthquake. Science 293, 1814-1818.

Suppe, J., 1985. Principles of Structural Geology. Prentice-Hall, New Jersey, 537 pp.

Takagi, H., 2001. Interactive evolutionary computation: Fusion of the capacities of EC optimization and human evaluation. Proceedings of the IEEE 89, 1275-1296.

Tarantola, A., 1987. Inverse Problem Theory. Elsevier, Amsterdam, 613 pp.

Thatcher, W., Foulger, G., Julian, B., Svarc, J., Quilty, E., Bawden, G., 1999. Present day deformation across the Basin and Range province, western United States. Science 282, 1714-1718. 